\title{
Intradural neurenteric cyst of the cervical spine misdiagnosed as a psychogenic disorder in a 7-year-old-child
}

\author{
Joji Mochida ${ }^{1}$, Shinya Yamada ${ }^{2}$, Eiren Toh $^{1}$, Masayoshi Shibata ${ }^{2}$ and Kazutoshi Oota ${ }^{1}$ \\ ${ }^{1}$ Department of Orthopaedic Surgery and ${ }^{2}$ Department of Neurosurgery, Tokai University, School of Medicine, \\ Bohseidai, Isehara, Kanagawa 259-11, Japan
}

\begin{abstract}
The patient is a 7-year-old Japanese female child with an intradural neurenteric cyst of the cervical spine. She had been misdiagnosed as having a psychogenic disorder due to the absence of neurological changes and bony anomalies. Subjective recovery followed the removal of the cyst.
\end{abstract}

Keywords: cervical neurenteric cyst; cervical spine; psychogenic disorder

\section{Introduction}

The number of patients reported with spinal cystic lesions has increased since the introduction of magnetic resonance imaging, and reports of children with cervical neurenteric cyst have been published.

\section{Case report}

A 7-year-old Japanese female presented to Tokai University Hospital with 6 months of progressive neck pain and limitation of motion. She had presented to another institution 5 months prior to admission to our hospital, and diagnosed with probable meningitis. Intravenous antibiotics were administered, although no cerebrospinal analysis was carried out. She was discharged from that institution following a remission of her symptoms. But they recurred in 7 days, and she presented to another national hospital and was diagnosed as having a psychogenic reaction in the absence of abnormal objective neurological changes despite having significant neck pain. The neck pain resolved after 2 or 3 days and there was subjective improvement in her condition. She had received psychotropic drugs such as chlorpromazine hydrochloride for 4 months. She then presented to our hospital with recurrent symptoms and was unable to sit up due to neck pain.

Initial physical examination showed a well-developed child with severe nuchal stiffness. The neurological examination revealed moderate resistance to neck flexion, but was otherwise normal. Her medical history was unremarkable. Laboratory data revealed a white cell count of 9200 cells/cu mm, normal C reactive protein, and an erythrocyte sedimentation rate of

Correspondence: J Mochida, MD, Department of Orthopaedic Surgery, Tokai University, School of Medicine, Bohseidai, Isehara, Kanagawa 259-11, Japan
$11 \mathrm{~mm} / \mathrm{h}$. Lumbar puncture at L4/5 yielded clear cerebrospinal fluid without cells, with $163 \mathrm{mg} / \mathrm{dl}$ protein, and normal glucose.

Plain radiographs revealed marked widening of the cervical spinal canal from $\mathrm{C} 3$ to $\mathrm{C} 6$, with an anteroposterior space of $19 \mathrm{~mm}$ at C4. Cervical magnetic resonance (MR) imaging revealed an anterior intradural extramedullary mass lesion from C3 to C5. Enhancement by Gd-DTPA was not observed, and the mass had a signal intensity similar to cerebrospinal fluid (Figure 1). Cranial MR imaging was unremarkable. Computerized tomographic myelography also demonstrated the mass lesion from $\mathrm{C} 3$ to $\mathrm{C} 5$ as a homogenic low density mass. The cervical spinal cord was posteriorly displaced. Computerized tomograms were also imaged 3, 6, and $24 \mathrm{~h}$ following subarachnoidal injection of $6 \mathrm{ml}$ contrast material, and the material did not flow into the lesion. Associated anomalies, such as multiple vertebral segmentation fusion, and anterior spina bifida, were not shown (Figure 2). This mass was therefore diagnosed as an intradural extramedullary cyst without communication to the subarachnoid space.

The patient had an en bloc suspended laminotomy from $\mathrm{C} 3$ to $\mathrm{C} 6$, with a hinge at the posterior ligamentous complex of the interspinous space between $\mathrm{C} 2$ and $\mathrm{C} 3$. Pulsation of the exposed dural sac was not observed. With the spinal cord medially displaced, the anterior cystic mass at C3 to C5 was visible. The mass contained a bluish fluid and measured about 3 by $2 \mathrm{~cm}$. The mass was loosely fixed to the inner surface of the dural sac or the anterior aspect of the cord. However, a stalk extending from the cyst through a defect in the dura was not detected (Figure 3). Removal of two-thirds of the cystic membrane resulted in alleviation of the spinal cord compression. The suspended laminae were replaced and sutured with nylon threads. Pathological 
examination of the cystic membrane revealed a cystic lesion lined by tall columnar nonciliated epithelium containing mucus, resembling intestinal epithelium, consistent with a neurenteric cyst (Figure 4). ${ }^{1}$

Postoperative course. The severe neck pain immediately disappeared postoperatively. Follow-up MR imaging studies at more than 3 years have revealed no cystic recurrence (Figure 5).

a

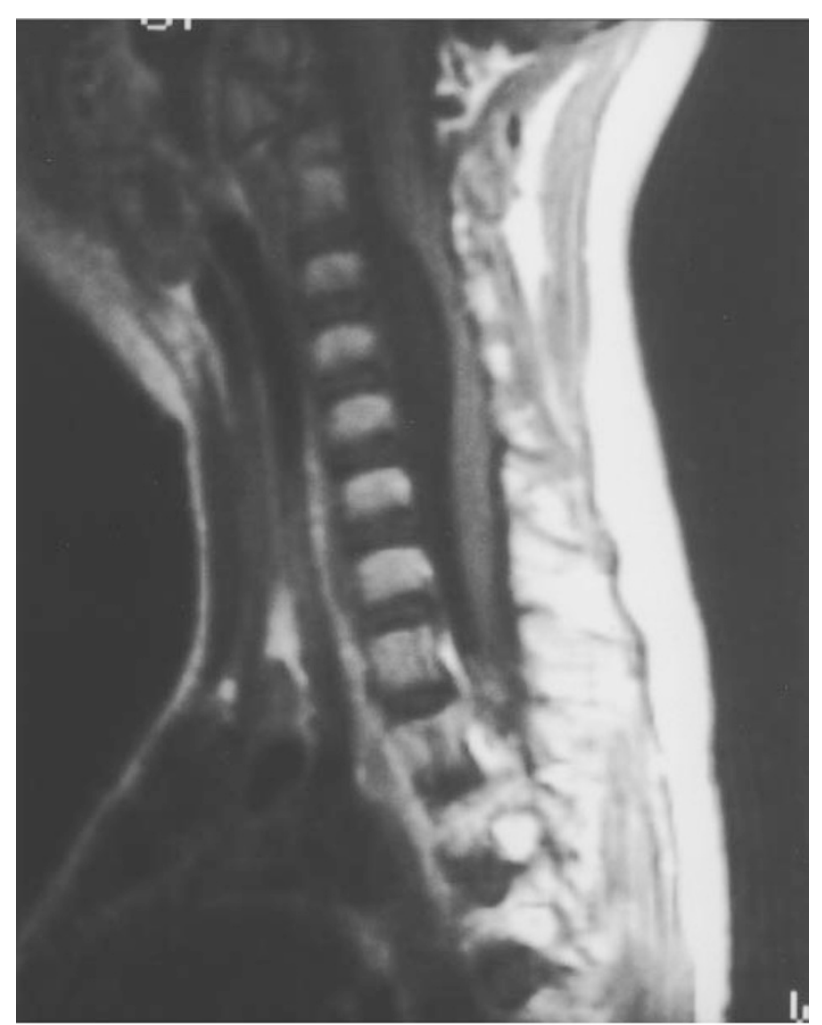

b

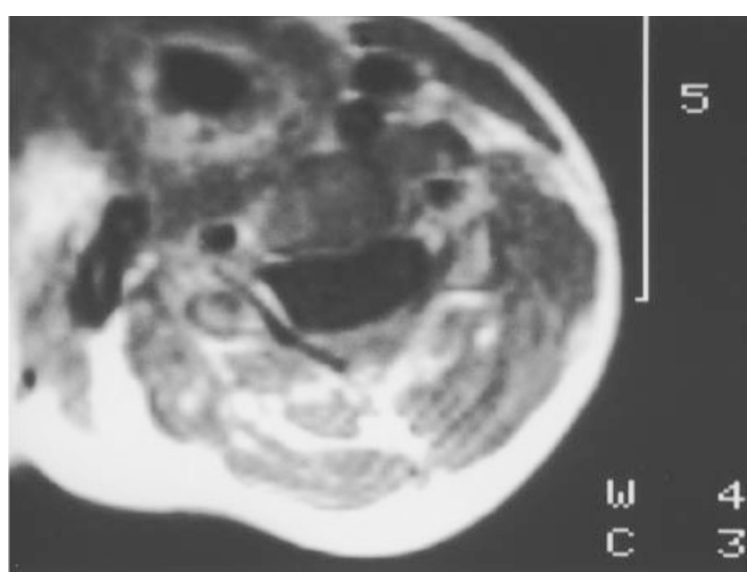

Figure 1 T1-weighted MR images enhanced by Gd-DTPA, sagittal (a) and axial (b) views, showing intradural extramedullary mass lesion from $\mathrm{C} 3$ to $\mathrm{C} 5$

\section{Discussion}

The pathogenesis of neurenteric cysts of the spinal cord remains uncertain, although it has been speculated to

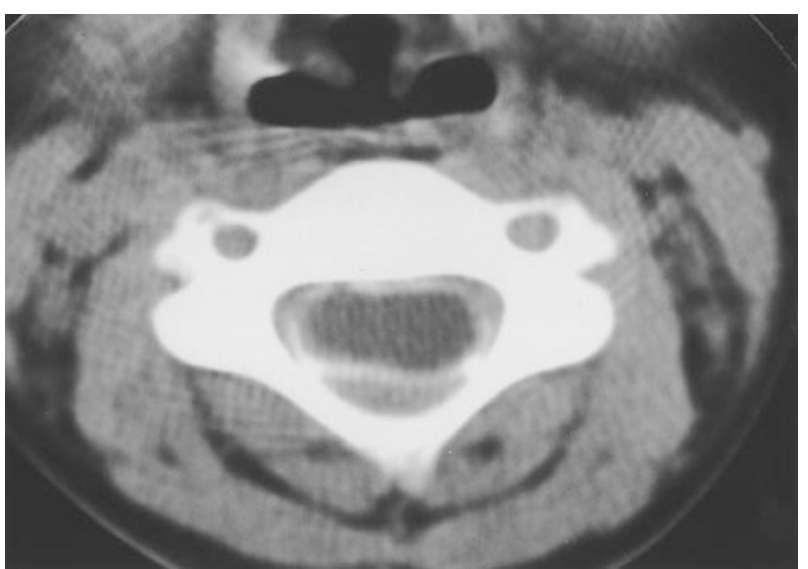

Figure 2 Computerized tomographic myelogram imaged $6 \mathrm{~h}$ after the subarachnoid injection of the contrast material revealing the posteriorly displaced cervical spinal cord and an anterior intradural extramedullary mass lesion without communication to the subarachnoid space. Associated bony anomalies were not shown

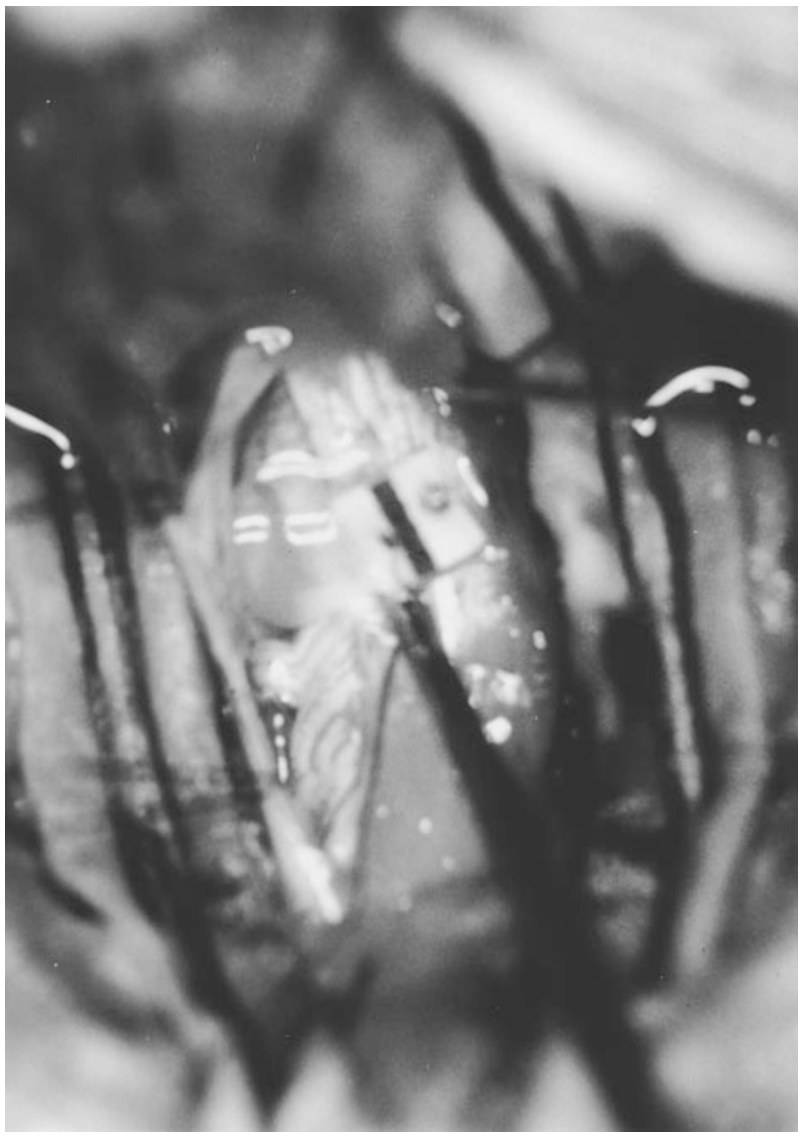

Figure 3 Intraoperative photograph showing the cystic mass 
be congenital in origin. ${ }^{2,3}$ The cysts result from dysgenesis occurring during the third week of embryogenesis, when the notochord develops and the neurenteric canal transitorily exits. The development of MR has increased the diagnosis of spinal neurenteric cysts. However, a true communication of the cyst with both the intestine and the spinal canal is rarely demonstrated on imaging. ${ }^{4-6}$

A particularly unusual feature of the patient reported here was severe neck pain without objective neurological findings, although most of the published cases were diagnosed with neurological abnormalities. This aspect of the case can be explained on the basis of the radiological studies. In general, the size of the cervical spinal canal in children is similar to that in adults by 3 years of age, and the development of the spinal cord follows subsequently. Asymptomatic mass lesions inside the spinal canal can occur rendering the absence of neurological findings in childhood unreliable. Despite the huge mass lesion in our patient, compression of neural structures was relatively mild and there was no significant neurological impairment. The neck pain was most probably due to involvement of the dorsal spinal nerve rootlets or roots of the middle cervical spine. The absence of contrast material inside the cyst suggested that there was no communication between the cyst and the subarachnoid space and an existence of higher intracystic pressure. Movement of the neck induces the change of the subarachnoidal pressure and the shift of the spinal cord toward anterior or posterior directions. An existence of the anterior neurenteric cyst may have a possibility to influence the normal passive movement of the spinal cord and the spinal nerve roots inside the spinal canal by the movement of the neck. This hypothesis is supported by fact that the neck pain and the meningismus sign were altered by different neck positions in this patient, being less severe when she was prone or supine.

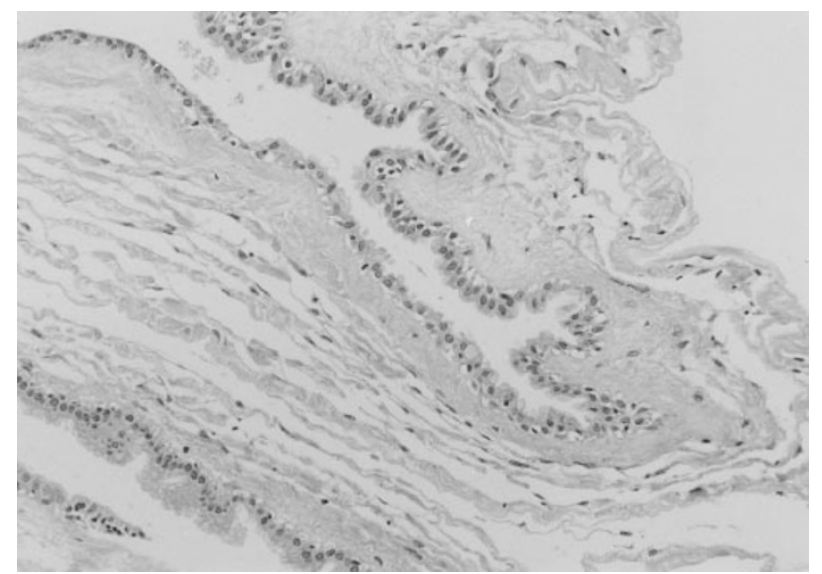

Figure 4 Photomicrograph of the cystic membrane demonstrating a cystic lesion lined by tall columnar, nonciliated epithelium containing mucus, resembling intestinal epithelium. (original magnification $\times 100$; hematoxylin and eosin)
Neurenteric cysts of the cervical spine in children are not very rare. In a review of the literature, most were diagnosed in the first decade of life. However, this 7-year-old patient could not be diagnosed as having the abnormal pathology for 6 months due to the absence of significant neurological impairment and the intermittent neck pain altered by different neck positions. The tragedy for this patient resulted from the pediatric and psychiatric staff in charge of her
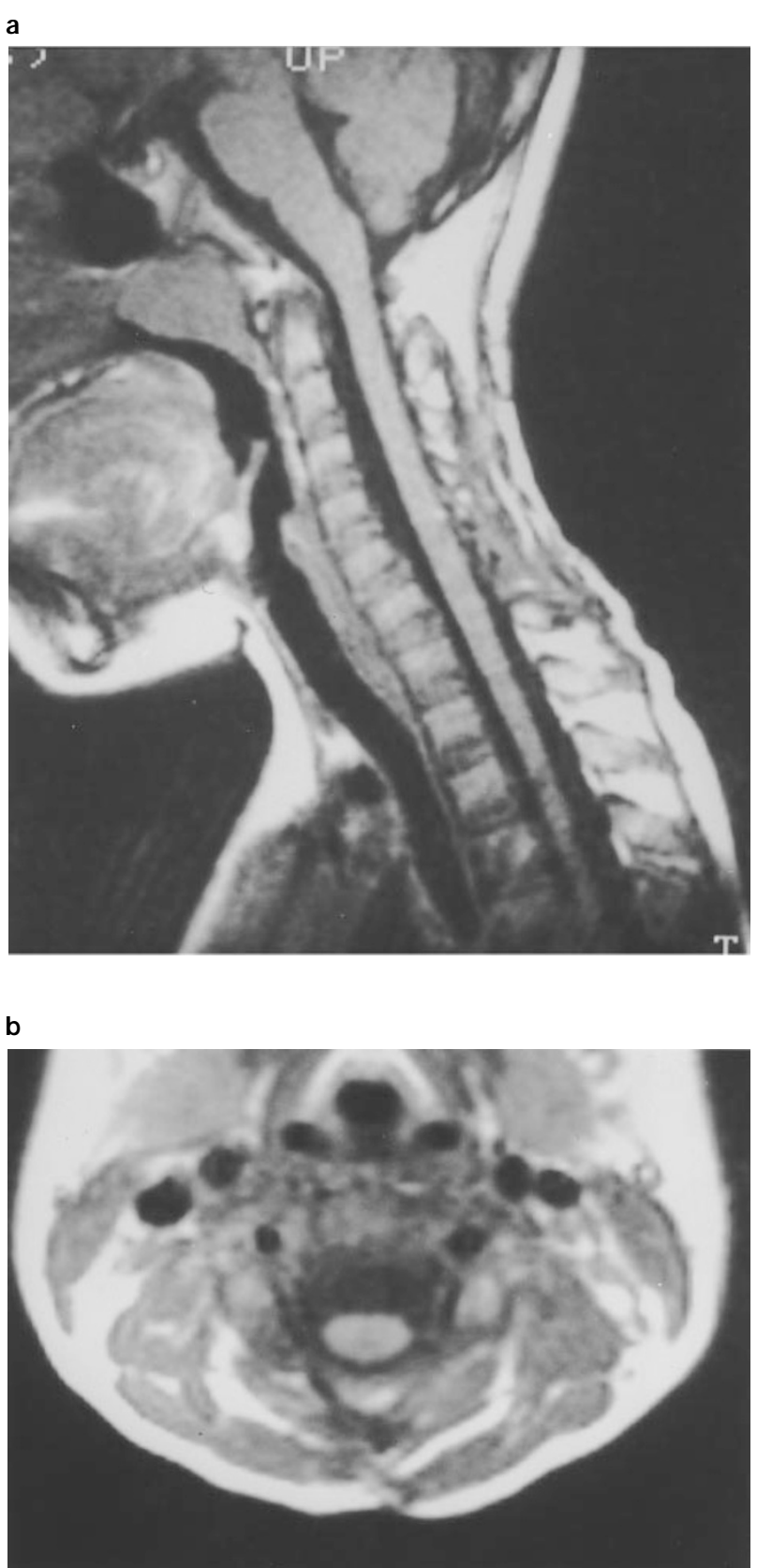

Figure 5 T1-weighted MR images, sagittal (a) and axial (b) views, at 37 months after the operation showing no recurrence of the cystic mass lesion 
initial treatment not appreciating the possible cause for her syndrome.

We should recognize that an intraspinal canal lesion in children cannot be denied even if there is no significant neurological impairment. Evaluation on MR imgages should be encouraged for children with persistent neck pain.

\section{Conclusions}

We present here a case of a neurenteric cyst of the cervical spine in a child with a rare episode of psychiatric treatment and have discussed the unusual presentation.

\section{References}

1 Matushima T, Fukui M, Egami H. Epithelial cells in a so-called intraspinal neurenteric cyst. A light and electron microscopic study. Surg Neurol 1985; 24: 656-660.

2 Alrabeeah A, Gillis DA, Giacomantonio M, Lau H. Neurenteric cysts. A spectrum. J Pediatr Surg 1988; 23: $752-754$.

3 Brooks BS et al. Neuroimaging features of neurenteric cysts: analysis of nine cases and review of literature. AJNR 1993; 14: $735-746$.

4 Fernandes ET et al. Neurenteric cyst. Surgery and diagnosis imaging. J Pediatr Surg 1993, 10: 304-306.

5 Kemp SS, Towbin RB. Pediatric case of the day. Radiographics 1992; 12: $1255-1257$.

6 Kantrowitz LR et al. Intraspinal neurenteric cyst containing gastric mucosa. CT and MRI findings. Pediatr Radiol 1986; 16: $324-327$. 